\title{
Characterising Wireless Sensor Motes for Space Applications
}

\author{
Tanya Vladimirova, Christopher P. Bridges, George Prassinos, \\ Xiaofeng Wu, Kawsu Sidibeh, David J. Barnhart, \\ Abdul-Halim Jallad, Jean R. Paul, Vaios Lappas, \\ Adam Baker*, Kevin Maynard* and Rodger Magness $†$ \\ Surrey Space Centre \\ Department of Electronic Engineering \\ University of Surrey \\ Guildford, UK GU2 $7 X H$ \\ \{ T.Vladimirova, C.P.Bridges, G.Prassinos, X.Wu, K.Sidibeh, D.Barnhart, A.Jallad, J.Paul, \\ V.Lappas\}@surrey.ac.uk \\ * Surrey Satellite Technology Limited (SSTL) \\ tEuropean Space Agency
}

\begin{abstract}
This paper is concerned with application of standard wireless COTS protocols to space. Suitability of commercially available wireless sensor mote kits for communication inside and between satellites is investigated. Spacecraft applications of motes are being considered and a set of requirements are identified. Selected mote kits are tested under various scenarios complying with spacecraft testing procedures. The paper details the results of the carried out functional, EMC/I, vibration, thermal and radiation tests.
\end{abstract}

Key words: Wireless sensor nodes, mote kits, satellites, wireless COTS protocols, distributed space systems

\section{Introduction}

Despite the fact that standard wireless commercialoff-the-shelf (COTS) protocols are widely used terrestrially they are not so popular in the space application domain yet. This paper is concerned with the application of standard wireless protocols for communication inside the satellite (intra-satellite communication and data gathering) and communication between satellites (inter-satellite communication).

Spacecraft platforms are highly complex systems. Harnessing and electrical interconnections of spacecraft components require a high level of assembly, integration and verification (AIV) effort. The use of wireless nodes inside the spacecraft for intra-satellite communication can potentially minimize the use of wires leading a significant reduction of the harness complexity, mass and AIV.

A system, where at least two or more satellites work cooperatively to perform a task, is defined as a distributed satellite system. The task is then referred to as a distributed space mission. There is a growing need to accomplish new types of missions in space where unusually large numbers of satellites are required. This scenario of a distributed satellite system consisting of a large number of satellites with inter-satellite communication links (ISL) is analogous to the concept of terrestrial wireless sensor networks (WSNs), where numerous devices, or nodes, are spread out over large areas to fulfil a particular monitoring/communication purpose.

Formation flying missions are of particular interest from the point of view of using wireless COTS protocols for inter-satellite connectivity due to the close proximity of the nodes in the network [1].

This paper presents the results of a feasibility study sponsored by the European Space Agency (ESA). The project looks into application of wireless sensor nodes on board small satellites manufactured by Surrey Satellite Technology Limited (SSTL). Suitability of COTS wireless sensor nodes, referred to as mote kits, for communication inside small spacecraft and between satellites is investigated. Selected mote kits are characterised for on-board use via functional, electromagnetic compatibility/interference (EMC/EMI), vibration, thermal and radiation testing.

The paper is structured as follows. Section 2 discusses the applications of wireless COTS protocols to small satellites including related EMC/EMI 
environmental considerations. Section 3 presents wireless mote kits description and comparison. Section 4 gives details of testing and presents test results. Section 5 concludes the paper.

\section{Application of Wireless COTS Protocols to Small Satellites}

Several intra-spacecraft applications are appropriate for wireless motes. We consider two such applications: telemetry gathering for housekeeping and use of motes to replace the on-board data bus.

The SSTL satellite platform currently uses the Controller Area Network (CAN) protocol as the onboard network for communication between subsystems. CAN has a limited data rate, requires wires and therefore becomes less efficient as the spacecraft complexity and size increases. Wireless nodes located in all subsystems of the spacecraft can be used as a replacement of the CAN bus.

"Strawman" mission scenarios requiring intersatellite communication which are of interest to SSTL and have the potential to demonstrate the advantages of wireless networks against current mission architectures were analysed [2].

\subsection{Wireless COTS Protocols Trade-off Analysis}

The wireless COTS protocols, which were investigated are ZigBee (IEEE 802.15.4) [3,4] and Bluetooth (IEEE 802.15.1) [5] for intra-satellite communication, and WiFi (IEEE 802.11) [6] and WiMax (IEEE 802.16) [7] for inter-satellite communications. We have come to the conclusion that WiFi or WiMax could be utilised for inter-satellite links while ZigBee could be used for intra-satellite links.

The criteria for the selection and recommendation of the wireless protocols for intra-satellite communication are: robustness of the protocol to fault and interference, support for many nodes, data rate, power requirement and easy connection to SSTL TCP/IP devices.

ZigBee is appropriate to be used where there is the need for mesh networking of sensor nodes within the spacecraft for monitoring and control. The CAN bus can transfer up to 1 Mbps but the existing CAN hardware offers a maximum rate of $32 \mathrm{Kbps}$, in which case the performance of IEEE 802.15.4 is acceptable for intra-spacecraft communications. Replacing CAN with a wireless mote system can lead to $80 \%$ mass savings, increased data rates and possible power saving. Requirements for additional communication path onboard with a higher rate data of above $1 \mathrm{Mbps}$ will need the use of UWB or IEEE 802.11.

An intermediate solution in the transition to wireless intra-satellite communication for CAN-based satellites could be realization of CAN over Bluetooth [8]. Several methods to convert Bluetooth to CAN and vice versa have been proposed for automotive applications [9]. A real-time operating system (OS), such as the TinyOS, could be employed to provide control capabilities in the converter. It is of interest to develop an on-board Bluetooth to CAN converter with TinyOS adopting the existing commercial solutions as a platform [10].

The criteria for the selection and recommendation of the wireless protocols for inter-satellite links are based on range, power requirements, networking features (especially MAC functions in close proximity links supporting many nodes) and finally support for mobility.

Although the IEEE 802.16e WiMAX standard is still to establish itself as a major player in the wireless data market, it provides some advantages, which are not available in other standards. For example, WiMAX features such as extensive support for QoS, high mobility, full native IP support, OFDMA and high physical layer efficiency are very valuable for intersatellite communications. However WiMax is fighting a battle against more established standards such as IEEE 802.11, including the up-and-coming IEEE 802.11n. With the emergence of Mobile WiMax in the near future, deployments that combine the two technologies (WiMAX and WiFi) can be constructed to take advantage of the strengths of both.

Wi-Fi supported by directional antennas and mesh network deployment could be used for close proximity links in formation flying missions. Extensions could be provided using WiMAX to cover last mile $(>10 \mathrm{~km})$ inter-satellite connectivity in LEO satellite constellations such as the SSTL DMC. It is believed that the IP core network at the basis of Wi-Fi and WiMAX will simplify networking with other IP technologies in future space networks.

The possibility of using ZigBee-Pro systems for up to $1.6 \mathrm{Km}$ range (20 $\mathrm{dBm}$ EIRP) at $250 \mathrm{Kbps}$ for sensor networking in inter-satellite applications could also be considered.

\subsection{Electromagnetic Compatibility/Interference}

Intra-satellite wireless communication is a subject to electromagnetic interference and radiation effects. The quality of wireless links suffers from time-varying channel degradations such as interference, flat-fading and frequency-selective fading. In addition to the natural sources of radiation electronic devices on board spacecraft produce radiation too. Internal frequencies and bandwidths of on-board equipment vary from a few hundred $\mathrm{Hz}$ to several GHz. Electromagnetic interference can lead to malfunctioning of the electronic devices on board spacecraft or even permanent damage. Especially that motes have no shielding, which makes them even more susceptible to EMI. Testing needs to be conducted to ensure correct operation of the various components when they operate together.

Wireless ISL communication utilizes several different frequencies and is a subject to interference and signal disruption from a broad spectrum of sources. The effects of noise and interference are different at 
different frequencies and can affect considerably the data link connectivity, reliability and data rates of the network. External interference can result in temporary or even permanent disruption of network connectivity and services, which can even result in mission failure.

NASA and ESA EMC requirements are within the specifications set by MIL-STD 461, 462 and 463. All NASA applications require attenuation of $60 \mathrm{~dB}$ or more, however SSTL spacecrafts EMC radiation is set to $40 \mathrm{~dB} \mu \mathrm{V} / \mathrm{m}$ for S-Band frequencies (circa $2 \mathrm{GHz}$ ).

Electromagnetic shielding is used to reduce the interference. The reduction depends on the shielding material, its thickness and the frequency.

\subsection{Environmental Operability and Survivability}

Wireless sensor mote solutions have not yet been considered for hostile conditions, specifically for those encountered in space [11]. A number of environmental hazards determine the operability and survivability of such nodes as described below:

- Mechanical (shock, vibration, acceleration): Fragile electronic structures may not be suitable for applications where excessive shock, vibration, and/or acceleration may exist.

- Atmospheric (corrosion, debris, vacuum): Corrosion is an issue for low-Earth orbit (LEO), industrial/chemical, and biomedical applications.

- Thermal (extremes, limited heat transfer): Thermal extremes and cycling are exacerbated in a vacuum, as thermal radiation is the only method available for heat transfer between space and a mote.

- Energetic (radiation, including charged particles): Extreme radiation conditions are experienced in space. High-energy charged particles are the cause of single-event effects (SEEs). A total dose hardness of 5-10 $\mathrm{Krad}(\mathrm{SiO} 2)$ is desirable for a multi-year mission in LEO, as the dose rate is on the order of $1 \mathrm{Krad}(\mathrm{SiO} 2)$ per year. Single-event hardness is also desirable, however determining hardness levels through testing is very expensive and is usually anticipated and mitigated through software and hardware design redundancy.

- Dynamic (free-fall orbit, high velocity mobility, attitude disturbance torques): Orbital velocity in LEO is approximately $7.5 \mathrm{~km} / \mathrm{s}$. Natural, but undesirable perturbations change the orbit over time. This factor must be fully understood, so key parameters like communication range can be selected properly. The freefall environment also presents unique challenges. The dominant effect is that objects in orbit "float" and change their orientation or "attitude" based on perturbations from solar pressure, gravity gradients, magnetic fields, and aerodynamic drag. This may not be an issue if the sensor technology does not have pointing requirements. However, if attitude control is required, solutions are very challenging at this scale.
Space debris is normally considered a hazard for satellites, but for a mission where thousands of objects (such as motes) are put in space, they become a big concern to other systems. The only realistic way to solve this problem is to confine these missions to LEO, where the orbital lifetime is very short, essentially making these missions disposable.

\section{Description and Comparison of Wireless Mote Kits}

A mote is an abbreviation for 'remote' node and refers to the individual units of sensing in a wireless sensor network. A typical mote architecture comprises the following basic components: a processor, transceiver and a sensing unit. Optional components of a mote are: a location finding system e.g. GPS and a mobiliser when movement of the motes is necessary.

There are two distinct types of low power, low data rate radios modules: narrowband and wideband radios. Enhanced modulation schemes found in wideband radios, such as spread spectrum (DSSS) and phase shift keying (O-QPSK), provide signal robustness to noise and interference. Narrowband radios typically operate at lower frequencies and lower data rates; wideband radios typically operate in the $2.4 \mathrm{GHz}$ band and provide higher data rates.

Due to the extreme mass and power constraints, most motes have limited processing capabilities with most motes using 8-bit processors, some using 16-bit processors and extremely few using a 32-bit processor. The processor is often used to implement the communications stack and do in-network data processing in order to achieve savings in bandwidth and therefore in power consumption.

Table 1 contains collated information on six mote kits, which were initially studied [12-16]. The requirements for the motes are determined by application: intra- and inter-satellite communications [2]. The commercial motes which have been selected for testing are: the TelosB motes from Crossbow [14], the BTNode from Art of Technology [15] and the High Powered Modules (HPM) from Jennic [16].

\section{Testing Results}

Testing aims to verify all important responses of the selected wireless motes and more specifically, how they operate under flight conditions on board a satellite in LEO. The tests are started from the least destructive functional tests in a lab environment to most destructive namely, thermal and radiation.

The functional tests comprise measurements of the power consumption under various modes of operation and wireless communication characteristics such as packet error rate (PER) as well as testing of ad-hoc networking functionality. The measured data are used to compare the mote kits and refer to after the more destructive tests such as thermal or radiation. 
Table 1. Mote Comparison Chart

\begin{tabular}{|c|c|c|c|c|c|c|c|}
\hline Mote Type & Mica2 & Mica-2 Dot & Telos & Eyes & \multicolumn{2}{|c|}{ BT Nodes } & Jennic \\
\hline \multicolumn{8}{|c|}{ Microcontroller } \\
\hline Type & \multicolumn{2}{|c|}{ Atmega128 } & \multicolumn{2}{|c|}{ TI MSP430 } & \multicolumn{2}{|c|}{ Atmega128 } & $\mathrm{SoC}$ \\
\hline Program memory (KB) & \multicolumn{2}{|c|}{128} & 48 & 60 & \multicolumn{2}{|c|}{128} & 64 \\
\hline RAM $(\mathrm{KB})$ & \multicolumn{2}{|c|}{4} & 10 & 2 & \multicolumn{2}{|c|}{4} & 96 \\
\hline Active Power (mW) & 8 & 33 & 3 & & \multicolumn{2}{|c|}{8} & \\
\hline Sleep Power $(\mathrm{uW})$ & 75 & 75 & 15 & & \multicolumn{2}{|c|}{75} & 37.8 \\
\hline Wakeup Time (us) & 180 & 180 & 6 & & \multicolumn{2}{|c|}{180} & \\
\hline \multicolumn{8}{|c|}{ Non-volatile Storage } \\
\hline Chip & \multicolumn{2}{|c|}{ AT45DB041B } & & & & & SoC \\
\hline Size (KB) & \multicolumn{2}{|c|}{512} & & & & & 128 \\
\hline \multicolumn{8}{|c|}{ Communication } \\
\hline Radio & \multicolumn{2}{|c|}{$\mathrm{CC} 1000$} & $\mathrm{CC} 2420$ & TR1001 & $\begin{array}{c}\mathrm{Zv} 400 \\
2\end{array}$ & $\mathrm{CC} 1000$ & $\begin{array}{c}\text { O-QPSK } \\
\text { Modem }\end{array}$ \\
\hline Data rate $(\mathrm{kbps})$ & \multicolumn{2}{|c|}{38.4} & 250 & & & 38.4 & 250 \\
\hline Receive power (mW) & \multicolumn{2}{|r|}{29} & 38 & & & 29 & 148.5 \\
\hline Transmit power at $0 \mathrm{dbm}(\mathrm{mW})$ & \multicolumn{2}{|r|}{42} & 35 & & & 42 & $\begin{array}{c}324 @ \\
18.5 \mathrm{dBm} \\
\end{array}$ \\
\hline \multicolumn{8}{|c|}{ Power Consumption and Mass } \\
\hline Minimum operation (V) & \multicolumn{2}{|c|}{2.7} & & & \multicolumn{2}{|c|}{0.85} & 2.7 \\
\hline Total Active power (mW) & 89 & 44 & 41 & & \multicolumn{2}{|c|}{102.3} & \\
\hline Mass (grams) & 18 & 3 & 23 & & \multicolumn{2}{|c|}{8} & $<2$ \\
\hline
\end{tabular}

The average power consumption results for the transmit, receive and sleep modes of operation of the three mote kits are presented in Table 2.

\section{Table 2. Average Power Consumption Results} (mW)

\begin{tabular}{|l|l|l|l|}
\hline Power Mode & Trasmit & Receive & Sleep \\
\hline Jennic HPM & 403.2 & 195.8 & 24.7 \\
\hline BTNode & 207.9 & 170.6 & 20.0 \\
\hline TelosB & 72.1 & 75.2 & 22.2 \\
\hline
\end{tabular}

To measure the long range capability of the Jennic High Power Modules, direct line of sight (LOS) connection is required over a long range. To achieve a long distance, a large park area (Stoke Park located in Guildford, UK) was used to get a LOS connection of around $865 \mathrm{~m}$. The packer error rate (RER) with and without acknowledgement return messages was measured. The test showed that even at these long distances, the packet error rate is very low, typically under $1 \%$.

\subsection{Electromagnetic Compatibility and Interference Testing}

This test describes the coexistence performance of the SSTL S-band transceiver when operating in an environment together with wireless motes. The potential interference of a mote with an S-Band receiver can be evaluated from the requirement that there should not be any interference from the mote with SSTL's receivers operating at a $2.06 \mathrm{GHz}$ frequency. This would require the motes to reject signals with frequencies outside the $20 \mathrm{MHz}$ bandwidth by at least $50 \mathrm{~dB}$.
Furthermore the transmitting sensor should not be operating at frequencies at the vicinity of the S-Band receiver. The equipment used includes a spectrum analyser (from 0 to $40 \mathrm{GHz}$ as measurements), a computer as data recording device, high gain double ridge horn antenna (of 24.2 by 13.6 opening) and transmitter-receiver pairs of Jennic, BTnodes and TelosB wireless motes. The transmitting mote was placed at a distance of more than $1 \mathrm{~m}$ in an anechoic chamber to allow measurement of the antenna's far field behaviour. The receiving mote was placed next to a high gain antenna, to record the radiated power.

The maximum power for the Jennic motes was found to be approximately $-53 \mathrm{dBm}$ at $2.42 \mathrm{GHz}$ when the resolution was set at $1 \mathrm{KHz}$ as shown in Figure 1. It was also observed that the spectrum analyser was functioning at its noise floor for frequencies outside the motes' bandwidth. Further the motes proved to have a rejection of more than $50 \mathrm{~dB}$ at its lowest operating frequency of $2.415 \mathrm{GHz}$. Thus we can conclude that the Jennic mote will not interfere with the SSTL's receivers.

The BTNodes were found to be operating far away from the SSTL's S-Band receiver, the filters also totally rejected all frequencies outside their operating range. Though there was no rejection of $50 \mathrm{~dB}$ the specifications were still met as shown in Figure 2. The TelosB motes also presented good rejection outside their bandwidth, and therefore would not cause any interference with the S-band receiver as shown in Figure 3. The TelosB motes use the TI MSP430 microcontroller, which has displayed good EMC in previous tests [17]. 


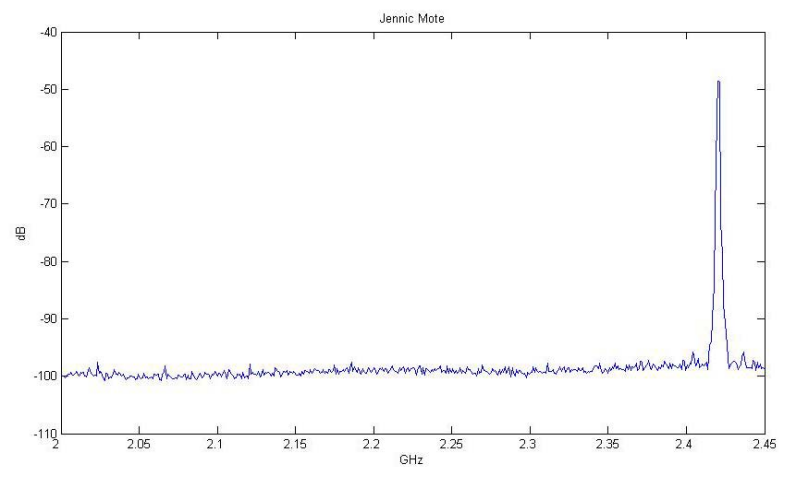

Figure 1. Jennic Mote EMC/I Test Result

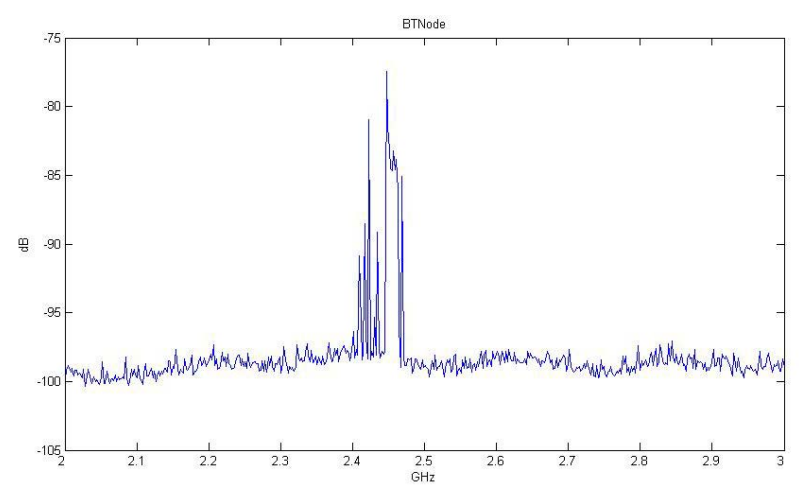

Figure 2. BTNode EMC/I Test Result

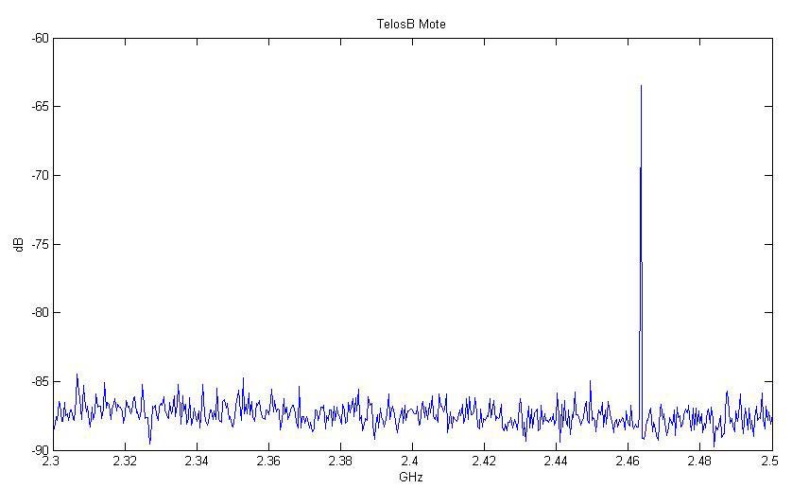

Figure 3. TelosB EMC/I Test Result

To conclude, the motes working in $2.4 \mathrm{GHz}$ presented good rejection capability outside their operating bandwidth as the spurious frequencies are too far away from SSTL's receivers to have an impact.

\subsection{Vibration Testing}

The objective of the random vibration tests is to verify the structure of the mote can withstand the flight limit loads multiplied by an appropriate safety factor of around $\pm 10 \%$ and to also find the resonant frequency of the device to ensure it does not fail during launch. If the launch process vibrates the mote at the resonant frequency, the higher amplitude oscillations could damage the mote, or affect the spacecraft structure itself.
Random vibration is where instantaneous magnitudes cannot be predicted and applied to the mote. This may be broad-band, covering a wide, continuous frequency range, or narrow band, covering a relatively narrow frequency range. This random vibration environment is specified in terms of acceleration power spectral density (PSD), plotted in terms of $\mathrm{g}^{2} / \mathrm{Hz}$ over a frequency range of 20 to 2000 $\mathrm{Hz}$, where $\mathrm{g}$ is the gravitational acceleration. For our test we are looking at tolerances of:

1. grms $(10 \%)$ to see the total acceleration over our emulated launch environment

2. PSD peak (typically $+3 \mathrm{~dB}$ ) from the control input to ensure that no resonances are excited that would affect the launcher

Test equipment included a $16 \mathrm{KW}$ shaker armature and table used to vibrate the nodes along 1 axis at any given time. Data recording of vibration results is collected via the accelerometers to a data recording computer. The computer has 8 channels; where 2 are used as control lines leaving 6 channels free for additional inputs. The test items were mounted to the vibration facility tables for excitation in the $\mathrm{X}, \mathrm{Y}$ and $\mathrm{Z}$ axis. The test fixture provides the interface between the test item and vibration table. Accelerometers are then installed onto the motes to collect vibration data. Vibration was then performed one axis at a time.

The test results are written in $\mathrm{g}^{2} / \mathrm{Hz}$ or Power Spectral Density (PSD) and plotted as a PSD curve for each axis as shown in Figures 4, 5 and 6.

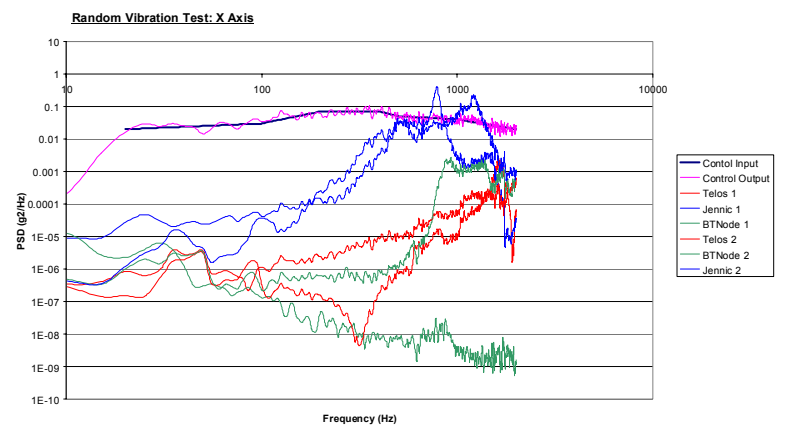

Figure 4. Vibration Test $\mathrm{X}$-axis

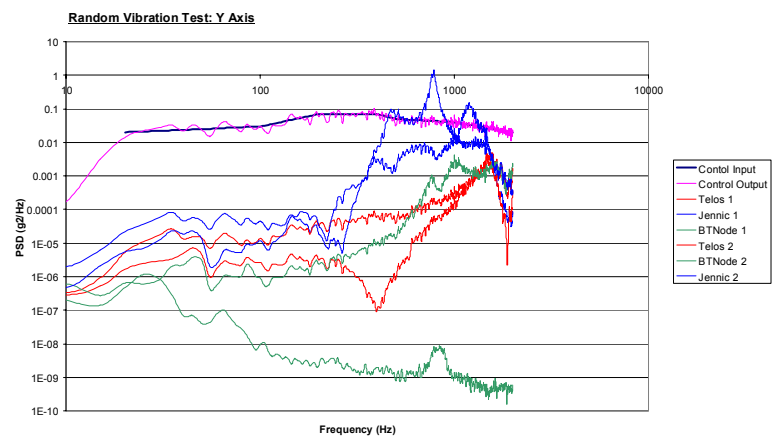

Figure 5. Vibration Test Y-axis 


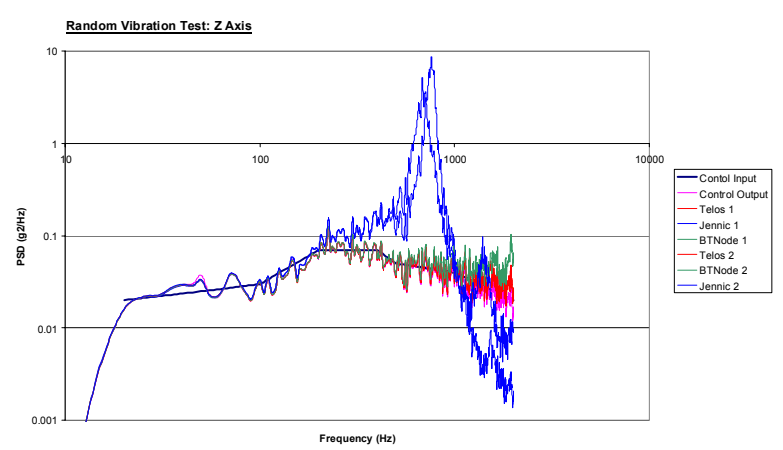

Figure 6. Vibration Test Z-axis

In the $\mathrm{x}$ axis (Figure 4), we find that the Jennic motes have two modes at high frequencies (namely at $790 \mathrm{~Hz}$ and $1,245 \mathrm{~Hz}$ ) that make them fail the PSD peak requirement. In the y axis (Figure 5), the Jennic motes fail at $785 \mathrm{~Hz}$ and $1,200 \mathrm{~Hz}$ for PSD peak requirement and one Jennic mote fails on the grms result, meaning that the average acceleration density is, over the whole band, not suitable for the launchers chosen in this test. In the $\mathrm{z}$ axis (Figure 6), we find that the armature is now shaking the motes in the worst case environment, where the boards are most likely to break (essentially, up and down). The loading here is at its fullest through the intermediate plate and adhesive. All the motes here fail in grms values and PSD peak requirements but due to the holding and fixing environment - variables that we had very little time to discuss and correct. The BTNodes and Jennic motes are one sided boards and had much glue to fix the parts to the intermediate plate - thus the amplification is greater. The TelosB motes however are double-sided and had only 4 points where glue was applied. This lack of contact with the plate acted to 'dampen' the structures vibration amplitude and this was seen in the results.

To conclude, this test showed three main outcomes:

- At lower frequencies less than $100 \mathrm{~Hz}$ (typically acoustic vibration), which are the areas of most concern for launch vehicles, there are no modes or resonances from any of the motes - making them suitable to be integrated to a launcher.

- The higher modes and resonances are very much dependant on the mass of the object and loading connected to. The Jennic motes here being a good example where the two resonance points were found and then amplified by the structure and holding mechanism. Careful design on how to hold/ implement the motes will dampen the effects of vibration and make them very suitable for launch.

- And finally, but not least, the motes survived this harsh testing environment (confirmed with functional tests).

\subsection{Thermal Testing}

Testing is typically carried out at a high level of integration (at least sub-system level) and not on each individual component of the payload. In this study test individual motes at a low level (only PCB's) are tested. The tests will be performed up to the qualification limits set by SSTL.

The thermal tests were carried out at the SSTL's facilities in Guildford, Surrey. The chamber has a temperature range: from $-40^{\circ} \mathrm{C}$ to $+80^{\circ} \mathrm{C}$ and 6,000 Cycles correspond to 1 year. The test duration is 2 days during which 4-8 cycles were carried out.

In the thermal chamber we tested eight (8) motes: two BTNodes motes, two Jennic motes, and two TelosB motes which where not powered up, one TelosB mote which was powered up to confirm correct wireless link operation while conducting the test and a powered Mica2 mote [14] which was used in order to monitor and record the condition within the chamber from a remote location.

In order to test power consumption of motes and perform an "on the fly" functional test a simple configuration using a power supply and a multi-meter was implemented. In order to accurately monitor the temperature within the chamber apart from using the chambers on-board reading we used Mica2 motes and, by forming a mesh network between our offices and the lab, were able to monitor the exact temperature within the chamber with up to two decimal digits accuracy.

The motes where tested for a thermal cycle between $-80^{\circ} \mathrm{C}$ and $+80^{\circ} \mathrm{C}$ in a period of fifty two (52) hours. This cycle, displayed in Figure 7, began from $+16.4^{\circ} \mathrm{C}$ up to $+40^{\circ} \mathrm{C}$ where the system was hold for thirty (30) minutes. Then the temperature was increased to $+80^{\circ} \mathrm{C}$ which was kept for twenty four (24) hours. The temperature after the duration of twenty four (24) hours was dropped to $-40^{\circ} \mathrm{C}$. The transition between $+80^{\circ} \mathrm{C}$ to $-40^{\circ} \mathrm{C}$ was done in sixty eight (68) minutes. The system was kept to $-40^{\circ} \mathrm{C}$ for twenty four (24) hours and then brought back to a temperature of $+40^{\circ} \mathrm{C}$ which was kept again for thirty (30) minutes.

After a visual inspection the devices did not appear to be damaged. A limited functional test on the TelosB was performed without any problems; examining three modes of operation of the motes, sleep, transmit and receive. During the tests switch-off and switch-on capability was successfully demonstrated at the low and high temperatures.

Functional tests are performed on the tested motes [2]. The average power results show that there is a minor degradation in power consumption. The Jennic motes displayed an increased power draw in the 'doze' and 'MCU running' modes suggesting that the internal microcontroller might have been damaged. The transmit power of the TelosB motes had also gone down, but not outside the specified range. 


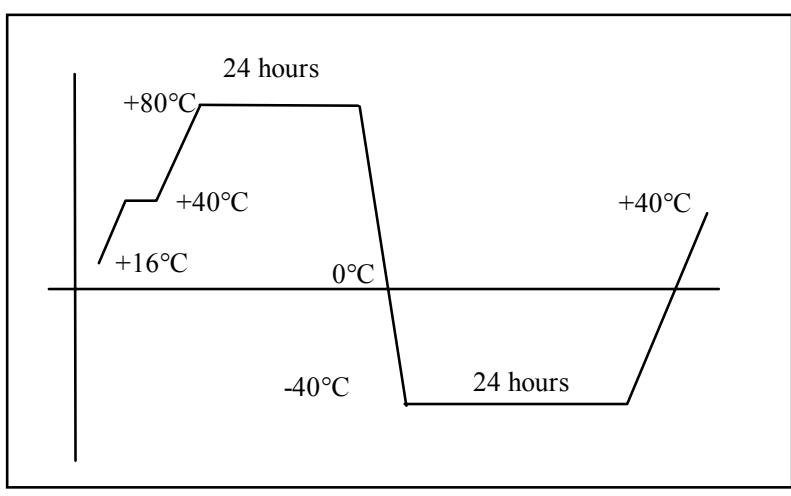

Figure 7. Thermal cycle diagram (not to scale)

\subsection{Radiation Testing}

Total ionizing dose radiation testing was performed at the National Physics Laboratory (NPL) Cobalt-60 Gamma-ray facility in the United Kingdom.

For the radiation testing, a dose rate of a total 30 $\mathrm{Krad}$ is examined. The dose rate is increased by increments of $5 \mathrm{Krad} / \mathrm{hr}$ from an initial dose rate of 0 Krad. The dose rate is measured and captured from several probes. During the process all the health features of the tested motes are recorded. The motes are also checked for correct transmission and operation.

On the day of testing, wiring was connected through safe entry points to the room to power the units. The radiation test setup is shown in Figure 8. Gaining wireless connectivity through the concrete and lead wall of radiation room to the safe zone was an important part of determining when the motes functionally failed. Connectivity was lost by the Jennic and BTNode motes throughout the test. The TelosB motes performed, overall the best with constant connectivity and no rise in current draw throughout the whole of the test to 30 Krads. No anomalies were found during the 6 hours of monitoring.

Power consumption results during the radiation test are shown in Figure 9. The results show that the TelosB and Jennic motes performed with nominal limits for the entire duration of the test except some connectivity anomalies. The BTNodes started to catastrophically fail at around 15 Krads where power consumption is latched up completely in a circuit. From the tests it was also seen that one BTNode functionally fails at around 8 Krads and the other at 15 Krads.

Following an annealing period of 48 hours at $23^{\circ} \mathrm{C}$, post-radiation testing of all the motes was carried out to determine the level of failures and functionality. After inspection, none of the mote kits could be reprogrammed. The Jennic and BTNodes allowed correct read back, as we would expect. That type of damage is typically from SEEs, meaning that none of the areas inside the memory has changed.

However, the mechanism which allows reprogramming fails in all the motes. This is internal to the device and cannot be fully investigated but damage from the gamma radiation is assumed to be the cause.
According to [18] it is often the peripheral circuitry that fails, such as the charge pump circuit used to add voltage to a particular line, and not the FLASH memory itself.

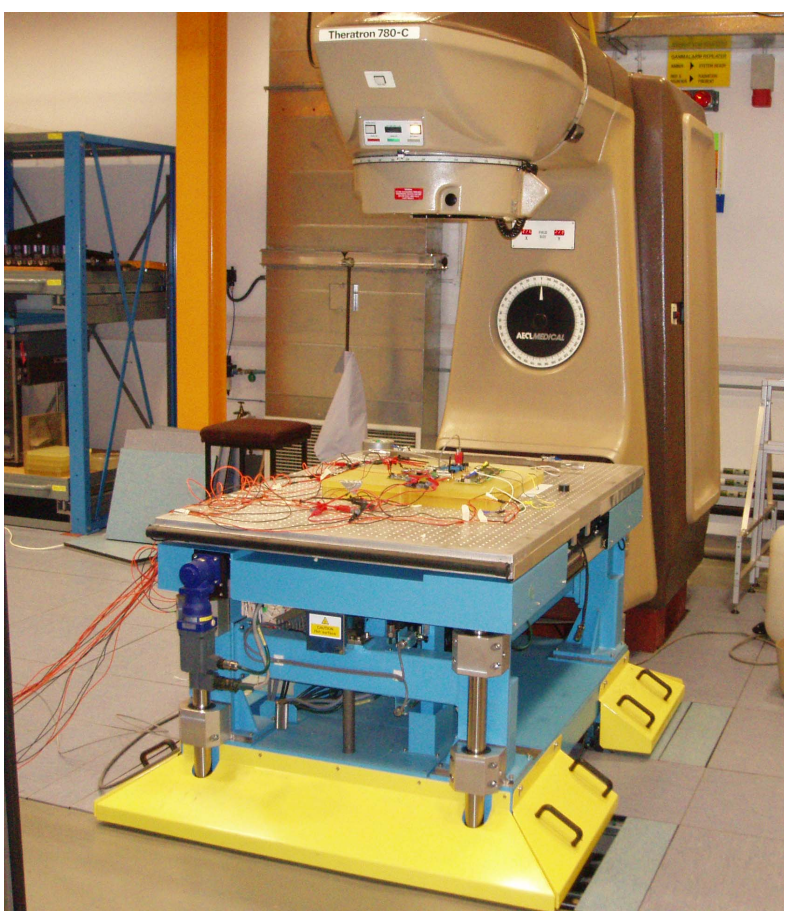

Figure 8. Radiation Test Setup

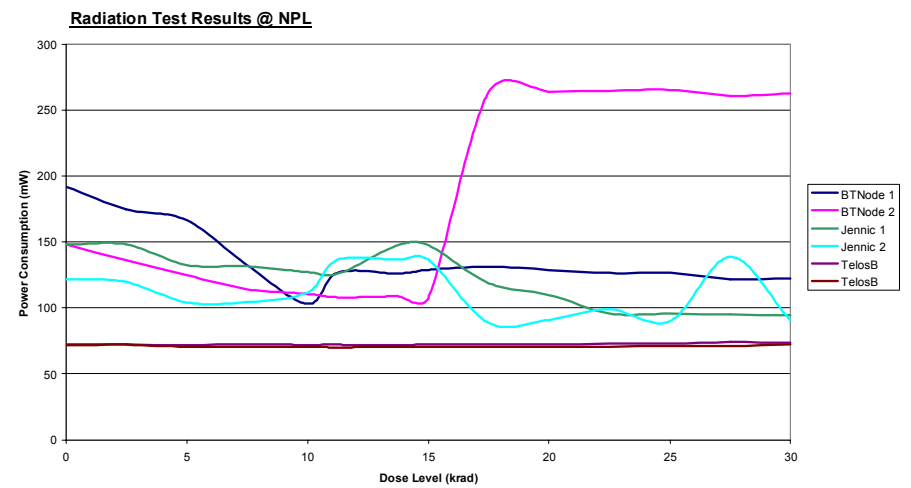

Figure 9. Power Consumption during Radiation Exposure

It can be noted that as all the motes are complete bought systems and a thorough test on every IC and device was not possible (e.g. on surface mount components).

\section{Conclusions}

This paper investigates standard wireless protocols and their application to space. We concluded that WiFi or WiMax (802.11 and 802.16 respectively) could be utilised for intersatellite links while ZigBee or 802.15.4 protocols could be used for intra-satellite links.

Commercially available mote kits are reviewed and applications for use on board small satellites 
identified. The first application is inter-satellite communications in close proximity formation flying satellite networks for which the Jennic high-power mote has been chosen primarily because it has the longest range found on mote technologies. The second application is intra-satellite data bus replacement for which the BTNode or TelosB motes where found to be satisfactory with their low power operation and $1 \mathrm{Mbps}$ capability with Bluetooth. Finally the third application is health monitoring and diagnostics for which we recommend using MicaA2 and Mica2dot motes.

Functional tests were performed to show that each mote kit had its merits and faults as follows:

- Jennic - good for long distance and mass but poor in power consumption and robust software development in harsh environments;

- BTNode - good for development and research into the Bluetooth L2P stack and routing possibilities but not as robust as other mote kits;

- TelosB - very good overall, excellent power consumption, robust design, small size, high data rate and considerably easy to program.

The EMC/I results were made to ensure that they did not interfere with SSTL transmitters or receivers. This was found to be true and had good rejection making all kits suitable for on-board operations. Thermal testing proved that all the mote kits are suitable for the space environment but future tests including a thermal and vacuum environment would be of greater interest. The vibration tests highlighted the need for a better structural method of implementing the motes in a satellite system, with adequate damping and mounting to avoid high amplification at the resonant frequencies. All the motes survived this test proving them to be durable and robust.

Exposure to radiation is harmful to mote kits, as they are made for terrestrial applications and radiation issues are not typically considered. However, despite failing during post radiation tests, the Jennic and TelosB motes performed functionally until reprogramming was required. For space applications memory protection from SEEs is needed and/or Flash memory types with heritage in space. It is also important to realise that the radiation tests were performed only on two nodes of each kit and there will be variation in component lots or batches making this test a very 'rough' estimate of the mote systems tested.

\section{Acknowledgements}

This research is sponsored by the European Space Agency.

\section{References}

1. C. I. Underwood, G. Richardson, J. Savignol, "SNAP-1: A Low Cost Modular COTS-Based Nano-Satellite Design, Construction, Launch and Early Operations Phase", Proceedings of AIAA/USU Conference on Small Satellites, Logan, UT, Aug. 13-16, 2001, SSC01-V-1a

2. Test Report, ESA Wireless Sensor Motes Study, Surrey Space Centre, University of Surrey, April 2007.

3. K. Sidibeh, T. Vladimirova, A. Baker, "Adapting the IEEE 802.11 Protocol for Inter-Satellite Links in LEO Constellations", Proceeding of Wireless for Space Applications Workshop, July 2006, ESTEC/ESA, Noordwijk, The Netherlands

4. ZigBee Specification, ZiBee Alliance (2005), June 2004, http://www.caba.org/standard/zigbee.html

5. IEEE 802.15.4 Part 15.4: Wireless Medium Access Control (MAC) and Physical Layer (PHY) Specifications for low-rate Wireless Personal Area Networks (LR-WPAN), October 2003, http://standards.ieee.org/ getieee802/download/802.15.4-2003.pdf

6. ANSI/IEEE 802.11 Standard, Wireless LAN Medium Access Control (MAC) and Physical Layer (PHY) Specifications, 1999

7. Mobile WiMAX Part 1: A Technical Overview and Performance Evaluation, WiMAX Forum, February 2006

8. R.D. Magness. "A comparison of CAN and Bluetooth- A study for Application of CAN over Bluetooth for Wireless Onboard Data Handling for a Spacecraft Sensor Network", Proceedings of DASIA'04, 2004

9. L. Fredriksson. "Bluetooth Automotive Diagnostics", http://www.kvaser.com/index.htm

10. M. Leopold. "Tiny Bluetooth Stack for TinyOS", Technical report, Dept of Computer Science, University of Copenhagen, April 2003, http://citeseer.ist.psu.edu/ leopold03tiny.html

11. D. J. Barnhart, T. Vladimirova, and M. N. Sweeting, "System-on-a-Chip Design of Self-Powered Wireless Sensor Nodes for Hostile Environments", Proceedings of the IEEE Aerospace Conference, Bozeman, MT, 2007, Paper 7.05.01.

12. Intel Motes: Advanced Sensor Network Platforms and Applications, Ralph M. Kling, Intel Corporation Research, Santa Clara, CA 95051, USA

13. Robert Shear, "Mesh, Motes and More", Wireless Net DesignLine, 05/30/2006, http://www.wirelessnetdesignline.com/

14. Crossbow Technology Inc., http://www.xbow.com/Products

15. BTNodes - A Distributed Environment for Prototyping Ad Hoc Networks, http://www.btnode.ethz.ch

16. Jennic Homepage, http://www.jennic.com

17. J. Delsing, J. Ekman, J. Johansson, S. Sundberg, M. Backstrom, M.; T. Nilsson, "Susceptibility of Sensor Networks to Intentional Electromagnetic Interference", Proceedings of 17th International Symposium on Electromagnetic Compatibility, pp. 172-175, Zurich, 2006.

18. D.N. Nguyen, "TID, SEE and Radiation Induced Failures in Advanced Flash Memories", Proceeding of Workshop for Radiation Effects Data, pp. 18- 23, 2003, IEEE 